MODELING, IDENTIFICATION AND CONTROL, 1991, VOL. 12, NO. 3, 107-115

doi:10.4173/mic.19913.1

\title{
A simplified algorithm of weighted generalized predictive adaptive control
}

\author{
WEI WANG $\dagger$ and ROLF HENRIKSEN $\ddagger$
}

Keywords: Predictive control, adaptive control.

In this paper a weighted generalized predictive controller and a simplified adaptive control algorithm using a multi-step cost-function with polynomial weighting are presented. A simulation example and a comparison of computational loads are also given in order to show the performance of the controller.

\section{Introduction}

Generalized predictive control (GPC) has enjoyed a growing attention in this last few years, see, e.g. Clarke et al. (1987a), De Keyser et al. (1988) and Garcia et al. (1989). The experimental studies and practical applications have demonstrated the robustness of GPC with respect to model-order and dead-time (Clarke and Mohtadi, 1989). Some interpretations and extensions of GPC are given by Clarke et al. (1987b) in which the role of the polynomials $P$ and $T$ are discussed respectively.

In this paper a weighted generalized predictive controller (WGPC) using a multistep cost function with the polynomial weighting is presented. The closed-loop system description with WGPC is derived. A simplified WGPC adaptive algorithm is also given based on the concatenation property of the optimal prediction.

\section{Weighted generalized predictive adaptive control}

Consider a plant described by the following CARIMA (controlled auto-regressive integrated moving-average) model

$$
A\left(z^{-1}\right) y(t)=B\left(z^{-1}\right) u(t-1)+C\left(z^{-1}\right) \omega(t) / \Delta
$$

where $A, B$ and $C$ are polynomials in the backward shift operator $z^{-1}$ with the degrees of $n_{a}, n_{b}$ and $n_{c}$ respectively. $A(0)=C(0)=1$ and $\Delta=1-z^{-1} \cdot y(t), u(t)$ and $\omega(t)$ are the output, input and disturbance respectively. $\mathscr{E}\left\{\omega(t) / \mathscr{F}_{t-1}\right\}=0, \mathscr{E}\left\{\omega(t)^{2} / \mathscr{F}_{t-1}\right\}=\sigma^{2}$, where $\mathscr{F}_{t-1}$ is the sub-sigma algebra generated by data up to time $t-1$.

The cost function has the form

$$
J=\mathscr{E}\left\{\sum_{j=1}^{N}\left(P\left(z^{-1}\right)\left(y(t+j)-y_{r}(t+j)\right)\right)^{2}+\sum_{j=1}^{N}\left(Q\left(z^{-1}\right) \Delta u(t+j-1)\right)^{2}\right\}
$$

Received 1 July 1991

$\dagger$ Department of Automatic Control, Northeast University of Technology, Shenyang, 110006, P.R. China. Currently on leave at Division of Engineering Cybernetics, The Norwegian Institute of Technology, 7034 Trondheim, Norway.

$\ddagger$ Division of Engineering Cybernetics, The Norwegian Institute of Technology, 7034 Trondheim, Norway. 
where $P$ and $Q$ are weighting polynomials with degrees of $n_{p}$ and $n_{q}$ respectively. $\left\{y_{r}(t)\right\}$ is a set-point sequence. $N$ is a prediction horizon. The polynomial $P$ is used here to penalize the over-shoot of the output whereas $Q$ is used to improve the dynamic behaviour of the input.

Define the auxiliary output $\psi(t)$ by

$$
\psi(t)=P\left(z^{-1}\right) y(t)
$$

According to Clarke and Gawthrop (1979), De Keyser and Van Cauwenberghe (1982) and using the Diophantine equation

$$
P\left(z^{-1}\right) C\left(z^{-1}\right)=A\left(z^{-1}\right) F_{j}\left(z^{-1}\right) \Delta+z^{-j} G_{j}\left(z^{-1}\right)
$$

the optimal j-step ahead prediction of $\psi(t)$ can be obtained as follows.

$$
C\left(z^{-1}\right) \psi^{\circ}(t+j / t)=G_{j}\left(z^{-1}\right) y(t)+B\left(z^{-1}\right) F_{j}\left(z^{-1}\right) \Delta u(t+j-1)
$$

In the following lemma we will present another possible representation of the optimal j-step ahead prediction of $\psi(t)$.

Lemma 2.1. For the system (2.1), if $\Delta u(t), \Delta u(t+1), \ldots$ are $\mathscr{F}_{t}$-measurable, the optimal j-step ahead prediction, $\psi^{\circ}(t+j / t)$, of $\psi(t)$ satisfies

$$
\psi^{\circ}(t+j / t)=D_{j}\left(z^{-1}\right) y(t)+B\left(z^{-1}\right) L_{j}\left(z^{-1}\right) \Delta u(t+j-1)+H_{j}\left(z^{-1}\right) \omega(t)
$$

where

$$
\psi^{\circ}(t+j / t)=\mathscr{E}\left\{\psi(t+j) / \mathscr{F}_{t}\right\}=\psi(t+j)-E_{j} \omega(t+j)
$$

$L_{j}, D_{j}, E_{j}$ and $H_{j}$ are here polynomials in $z^{-1}$ and satisfy uniquely the equations

$$
\begin{aligned}
& P\left(z^{-1}\right)=A\left(z^{-1}\right) L_{j}\left(z^{-1}\right) \Delta+z^{-j} D_{j}\left(z^{-1}\right) \\
& C\left(z^{-1}\right) L_{j}\left(z^{-1}\right)=E_{j}\left(z^{-1}\right)+z^{-j} H_{j}\left(z^{-1}\right)
\end{aligned}
$$

where

$$
\begin{gathered}
L_{j}\left(z^{-1}\right)=l_{0}+l_{1} z^{-1}+\ldots+l_{j-1} z^{-j+1} \\
D_{j}\left(z^{-1}\right)=d_{0}^{j}+d_{1}^{j} z^{-1}+\ldots+d_{w}^{j} z^{-w}, \quad w=\max \left(n_{a}, n_{p}-j\right) \\
E_{j}\left(z^{-1}\right)=e_{0}+e_{1} z^{-1}+\ldots+e_{j-1} z^{-j+1} \\
H_{j}\left(z^{-1}\right)=h_{0}^{j}+h_{1}^{j} z^{-1}+\ldots+h_{n_{c}-1}^{j} z^{-n_{c}+1}
\end{gathered}
$$

Proof. Multiplying (2.1) by $L_{j} \Delta$ and using (2.3) and (2.4) we obtain

$$
\psi(t+j)=D_{j} y(t)+B L_{j} \Delta u(t+j-1)+H_{j} \omega(t)+E_{j} \omega(t+j)
$$

where the operator $z^{-1}$ is omitted to simplify the notation. Now define

$$
\psi^{\circ}(t+j / t)=\psi(t+j)-E_{j} \omega(t+j)
$$

From (2.5) it follows that

$$
\psi^{\circ}(t+j / t)=D_{j} y(t)+B L_{j} \Delta u(t+j-1)+H_{j} \omega(t)
$$

which obviously is $\mathscr{F}_{t}$-measurable and hence

$$
\psi^{\circ}(t+j / t)=\mathscr{E}\left\{\psi^{\circ}(t+j / t) / \mathscr{F}_{t}\right\}=\mathscr{E}\left\{\psi(t+j)-E_{j} \omega(t+j) / \mathscr{F}_{t}\right\}=\mathscr{E}\left\{\psi(t+j) / \mathscr{F}_{t}\right\}
$$


Therefore,

$$
\mathscr{E}\left\{\left(\psi(t+j)-\psi^{\circ}(t+j / t)\right)^{2}\right\}=\sum_{i=0}^{j-1} e_{i}^{2} \sigma^{2}
$$

which establishes the optimality of $\psi^{\circ}(t+j / t)$.

Using

$$
B\left(z^{-1}\right) L_{j}\left(z^{-1}\right)=G_{j}\left(z^{-1}\right)+z^{-j} S_{j}\left(z^{-1}\right)
$$

where

$$
\begin{aligned}
& G_{j}\left(z^{-1}\right)=g_{0}+g_{1} z^{-1}+\ldots+g_{j-1} z^{-j+1} \\
& S_{j}\left(z^{-1}\right)=s_{0}^{j}+s_{1}^{j} z^{-1}+\ldots+s_{n_{b}-1}^{j} z^{-n_{b}+1}
\end{aligned}
$$

We can write $\psi^{\circ}(t+j / t)$ as

$$
\psi^{\circ}(t+j / t)=D_{j} y(t)+G_{j} \Delta u(t+j-1)+S_{j} \Delta u(t-1)+H_{j} \omega(t)
$$

Let $f(t+j)$ be the part of $\psi^{\circ}(t+j / t)$ composed of signals which are known at time $t$, i.e.

$$
f(t+j)=D_{j} y(t)+S_{j} \Delta u(t-1)+H_{j} \omega(t)
$$

Then the equation (2.7) can be written in the vector form

where

$$
\psi^{\circ}=\mathbf{G u}+\mathbf{f}
$$

$$
\begin{aligned}
\psi^{\circ} & =\left[\psi^{\circ}(t+1 / t), \ldots, \psi^{\circ}(t+N / t]^{\mathrm{T}}\right. \\
\mathbf{u} & =[\Delta u(t), \ldots, \Delta u(t+N-1)]^{\mathrm{T}} \\
\mathbf{f} & =[f(t+1), \ldots, f(t+N)]^{\mathrm{T}}
\end{aligned}
$$

whereas the matrix $\mathbf{G}$ is the lower-triangular of dimension $N \times N$ and of the form

$$
\mathbf{G}=\left[\begin{array}{cccc}
g_{0} & & & \\
g_{1} & g_{0} & & \\
\cdots & & & \\
g_{N-1} & g_{N-2} & \cdots & g_{0}
\end{array}\right]
$$

In order to split $Q \Delta u(t+j-1)$ in (2.2) into the two subsequences $\Delta u(t+N-1), \ldots, \Delta u(t)$ and $\Delta u(t-1), \ldots, \Delta u\left(t-n_{q}\right)$, the polynomial $Q$ is written as

where

$$
Q\left(z^{-1}\right)=\bar{Q}_{j}\left(z^{-1}\right)+z^{-j} Q_{j}\left(z^{-1}\right)
$$

$$
\begin{aligned}
& \bar{Q}_{j}\left(z^{-1}\right)= \begin{cases}q_{0}+q_{1} z^{-1}+\ldots+q_{j-1} z^{-j+1} & \left(j \leqslant n_{q}\right) \\
q_{0}+q_{1} z^{-1}+\ldots+q_{n_{q}} z^{-n_{q}} & \left(j>n_{q}\right)\end{cases} \\
& Q_{j}\left(z^{-1}\right)= \begin{cases}q_{j}+q_{j+1} z^{-1}+\ldots+q_{n_{q}} z^{-n_{q}+j} & \left(j \leqslant n_{q}\right) \\
0 & \left(j>n_{q}\right)\end{cases}
\end{aligned}
$$

Define

$$
\begin{aligned}
\boldsymbol{\Phi} & =[Q \Delta u(t), \ldots, Q \Delta u(t+N-1)]^{\mathrm{T}} \\
Q^{\prime} & =\left[Q_{1}, \ldots, Q_{N}\right]^{\mathrm{T}} \\
\mathbf{z} & =Q^{\prime} \Delta u(t-1)
\end{aligned}
$$


From (2.13) we then have

$$
\boldsymbol{\Phi}=\overline{\mathbf{Q}} \mathbf{u}+\mathbf{z}
$$

where the matrix $\overline{\mathbf{Q}}$ is also lower-triangular of dimension $N \times N$, viz.

$$
\overline{\mathbf{Q}}=\left[\begin{array}{cccc}
q_{0} & & & \\
q_{1} & q_{0} & & \\
\ldots & & & \\
q_{N-1} & q_{N-2} & \ldots & q_{0}
\end{array}\right]
$$

Note that $q_{n_{q}+1}, \ldots, q_{N-1}$ in (2.16) are zero if $n_{q}<N-1$. Define

$$
\mathbf{w}=\left[P y_{r}(t+1), \ldots, P y_{r}(t+N)\right]^{\mathrm{T}}
$$

and the expectation of the cost function (2.2) can be written as

$$
J=\left(\psi^{\circ}-\mathbf{w}\right)^{\mathrm{T}}\left(\psi^{\circ}-\mathbf{w}\right)+\Phi^{\mathrm{T}} \Phi
$$

The minimization of $J$ with respect to $u$ results in

$$
\mathbf{u}=\left(\mathbf{G}^{\mathrm{T}} \mathbf{G}+\overline{\mathbf{Q}}^{\mathrm{T}} \overline{\mathbf{Q}}\right)^{-1}\left[\mathbf{G}^{\mathrm{T}}(\mathbf{w}-\mathbf{f})-\overline{\mathbf{Q}}^{\mathrm{T}} \mathbf{z}\right]
$$

Note that the first element of $u$ is $\Delta u(t)$ and according to Clarke $e t$ al. (1987a) the receding horizon control $u(t)$ is given by

$$
u(t)=u(t-1)+g^{\mathrm{T}}\left[\mathbf{G}^{\mathrm{T}}(\mathbf{w}-\mathbf{f})-\mathbf{Q}^{\mathrm{T}} \mathbf{z}\right]
$$

where $g^{\mathrm{T}}$ is the first row of $\left(\mathbf{G}^{\mathrm{T}} \mathbf{G}+\overline{\mathbf{Q}}^{\mathrm{T}} \overline{\mathbf{Q}}\right)^{-1}$.

In order to avoid singularity of $\left(\mathbf{G}^{\mathrm{T}} \mathbf{G}+\overline{\mathbf{Q}}^{\mathrm{T}} \mathbf{Q}\right)$ and to reduce the computational load for a large output horizon, the technique of imposing a control horizon $N_{u}\left(N_{u}<N\right)$ can be used here, i.e. when $j>N_{u}$, we put $\Delta u(t+j-1)=0$. Therefore, in this case the vector u in (2.10) is changed into

$$
\mathbf{u}=\left[\Delta u(t), \Delta u(t+1), \ldots, \Delta u\left(t+N_{u}-1\right)\right]^{\mathrm{T}}
$$

and the dimensions of matrices $\mathbf{G}, \overline{\mathbf{Q}}$ and $\mathbf{G}^{\mathrm{T}} \mathbf{G}+\overline{\mathbf{Q}}^{\mathrm{T}} \mathbf{Q}$ are changed into $N \times N_{\mathrm{u}}, N \times N_{u}$ and $N_{u} \times N_{u}$ respectively.

\section{Closed-loop system}

In this section we will derive the closed-loop system equation when control law (2.18) is used. Define

$$
\mathbf{r}^{\mathrm{T}}=\left[r_{1}, r_{2}, \ldots, r_{N}\right]
$$

as the first row of $\left(\mathbf{G}^{\mathrm{T}} \mathbf{G}+\overline{\mathbf{Q}}^{\mathrm{T}} \mathbf{Q}\right)^{-1}$ and $\mathbf{v}^{\mathrm{T}}$ as the first row of $\left(\mathbf{G}^{\mathrm{T}} \mathbf{G}+\overline{\mathbf{Q}}^{\mathrm{T}} \overline{\mathbf{Q}}\right)^{-1} \mathbf{Q}^{\mathrm{T}}$. Also define

$$
\begin{aligned}
S & =\left[S_{1}, \ldots, S_{N}\right]^{\mathrm{T}} \\
D & =\left[D_{1}, \ldots, D_{N}\right]^{\mathrm{T}} \\
H & =\left[H_{1}, \ldots, H_{N}\right]^{\mathrm{T}}
\end{aligned}
$$

From (2.9) and (2.11) we have

$$
\mathbf{f}=D y(t)+S \Delta u(t-1)+H \omega(t)
$$


From (3.1) and (2.14) the control law (2.18) is written as

$$
\Delta u(t)=\mathbf{r}^{\mathrm{T}}(\mathbf{w}-D y(t)-H \omega(t))-\left(\mathbf{r}^{\mathrm{T}} S+\mathbf{v}^{\mathrm{T}} Q^{\prime}\right) \Delta u(t-1)
$$

Define

$$
\begin{gathered}
M=\mathbf{r}^{\mathrm{T}} S+\mathbf{v}^{\mathrm{T}} Q^{\prime} \\
R\left(z^{-1}\right)=r_{N}+r_{N-1} z^{-1}+\ldots+r_{1} z^{-N+1}
\end{gathered}
$$

We thus can write the control law in the form

$$
\Delta u(t)=\left(R P y_{r}(t+N)-\mathbf{r}^{\mathrm{T}} D y(t)-\mathbf{r}^{\mathrm{T}} H \omega(t)\right) /\left(1+z^{-1} M\right)
$$

Substituting (3.4) into (2.1) yields the closed-loop system

$$
T\left(z^{-1}\right) y(t)=B\left(z^{-1}\right) R\left(z^{-1}\right) P\left(z^{-1}\right) y_{r}(t+N-1)+C^{\prime}\left(z^{-1}\right) \omega(t)
$$

where the characteristic polynomial $T$ is given by

$$
T=A \Delta\left(1+z^{-1} M\right)+z^{-1} B \mathrm{r}^{\mathrm{T}} D
$$

whereas

$$
C^{\prime}=C\left(1+z^{-1} M\right)-z^{-1} B \mathbf{r}^{\mathrm{T}} H
$$

We can see from (3.5) that the system eliminates the steady-state output error in a natural way. From (2.3) we know that $D_{j}(1)=P(1)(j=1,2, \ldots, N)$, and from (3.3) and (3.6) we thus have

$$
T(1)=B(1) \sum_{i=1}^{N} r_{i} D_{i}(1)=B(1) P(1) \sum_{i=1}^{N} r_{i}=B(1) P(1) R(1)
$$

The stability of the closed-loop system depends on the location of the roots of the characteristic polynomial $T$. It is clear that the polynomials $P$ and $Q$ in addition to the design parameters $N$ and $N_{u}$ affect the roots of the polynomial $T$. The use of polynomials $P$ and $Q$ therefore increases the amount of the tuning knobs which gives the user a wider set of choices in the design of the system performance.

\section{A simplified WGPC adaptive algorithm}

Since the calculational load of GPC control law in adaptive case is quite heavy, a simplification of the algorithm is important for industrial control applications. A simplified algorithm for GPC control law was suggested by using a cost function without $P$ and $Q$ weighting polynomials (Favier, 1987). In this section a simplified WGPC adaptive algorithm will be derived by using the concatenation property stated in the following lemma.

Lemma $4.1 \cdot \psi^{\circ}(t+j / t)$ satisfies the following equation

$$
\begin{aligned}
\psi^{\circ}(t+j / t)= & -\alpha_{1} \psi^{\circ}(t+j-1 / t)-\ldots-\alpha_{j-1} \psi^{\circ}(t+1 / t) \\
& -\alpha_{j} \psi(t)-\ldots-\alpha_{n_{a}+1} \psi\left(t+j-n_{a}-1\right) \\
& +\beta\left(z^{-1}\right) \Delta u(t+j-1)+\frac{1}{p_{0}} C_{j}\left(z^{-1}\right)\left(\psi(t)-\psi^{\circ}(t / t-1)\right)
\end{aligned}
$$

where $\alpha_{j}\left(j=1,2, \ldots, n_{a}+1\right)$ satisfy

$$
A\left(z^{-1}\right) \Delta=1+\alpha_{1} z^{-1}+\ldots+\alpha_{n_{a}+1} z^{-n_{a}-1}
$$


Furthermore, $\beta\left(z^{-1}\right)$ and $C_{j}\left(z^{-1}\right)$ satisfy

where

$$
\begin{gathered}
\beta\left(z^{-1}\right)=B\left(z^{-1}\right) P\left(z^{-1}\right)=\beta_{0}+\beta_{1} z^{-1}+\ldots+\beta_{n_{b}+n_{p}} z^{-n_{b}-n_{p}} \\
C\left(z^{-1}\right) P\left(z^{-1}\right)=\bar{C}_{j}\left(z^{-1}\right)+q^{-j} C_{j}\left(z^{-1}\right)
\end{gathered}
$$

$$
\begin{aligned}
\bar{C}_{j}\left(z^{-1}\right) & = \begin{cases}\bar{c}_{0}+\bar{c}_{1} z^{-1}+\ldots+\bar{c}_{j-1} z^{-j+1} & (j \leqslant m) \\
\bar{c}_{0}+\bar{c}_{1} z^{-1}+\ldots+\bar{c}_{m} z^{-m} & (j>m)\end{cases} \\
C_{j}\left(z^{-1}\right) & = \begin{cases}\bar{c}_{j}+\bar{c}_{j+1} z^{-1}+\ldots+\bar{c}_{m} z^{-m+j} & (j \leqslant m) \\
0 & (j>m)\end{cases}
\end{aligned}
$$

and where $m=n_{\mathrm{c}}+n_{p}$.

Proof. Multiplying (2.1) by $P \Delta$ yields

Using (4.2) we have

$$
A\left(z^{-1}\right) \Delta \psi(t)=\beta\left(z^{-1}\right) \Delta u(t-1)+C\left(z^{-1}\right) P\left(z^{-1}\right) \omega(t)
$$

$$
A\left(z^{-1}\right) \Delta \psi(t+j)=\beta\left(z^{-1}\right) \Delta u(t+j-1)+\bar{C}_{j}\left(z^{-1}\right) \omega(t+j)+C_{j}\left(z^{-1}\right) \omega(t)
$$

According to lemma 7.4.2. in Goodwin and $\operatorname{Sin}(1984)$ and taking expectations given $\mathscr{F}_{t}$ we obtain

$$
\begin{aligned}
\psi^{\circ}(t+j / t)= & -\alpha_{1} \psi^{\circ}(t+j-1 / t)-\ldots-\alpha_{j-1} \psi^{\circ}(t+1 / t) \\
& -\alpha_{j} \psi(t)-\ldots-\alpha_{n_{a}+1} \psi\left(t+j-n_{a}-1\right)+\beta\left(z^{-1}\right) \Delta u(t+j-1) \\
& +\bar{c}_{j} \mathscr{E}\left\{\omega(t) / \mathscr{F}_{t}\right\}+\ldots+\bar{c}_{m} \mathscr{E}\left\{\omega(t+j-m) / \mathscr{F}_{t}\right\}
\end{aligned}
$$

From lemma 2.1 we know that

$$
\omega(t)=\frac{1}{p_{0}}\left(\psi(t)-\psi^{\circ}(t / t-1)\right)
$$

which shows that $\omega(t)$ is $\mathscr{F}_{t}, \mathscr{F}_{t+1}, \ldots$ measurable, (4.1) then follows from (4.3)-(4.5).

The implication of the lemma above is that the optimal j-step ahead prediction can be obtained from optimal one-step ahead prediction. This can be seen by writing (4.1) for $j=1,2, \ldots$ as follows.

and so on, where

$$
\begin{aligned}
& \psi^{\circ}(t+1 / t)=\beta_{0} \Delta u(t)+f^{\prime}(t+1) \\
& \psi^{\circ}(t+2 / t)=\left[\beta_{1}-\alpha_{1} \beta_{0}\right] \Delta u(t)+\beta_{0} \Delta u(t+1)+f^{\prime}(t+2)
\end{aligned}
$$

$$
\begin{aligned}
f^{\prime}(t+1)= & -\alpha_{1} \psi(t)-\ldots-\alpha_{n_{a}+1} \psi\left(t-n_{a}\right)+z\left[\beta\left(z^{-1}\right)-\beta_{0}\right] \Delta u(t-1) \\
& +\frac{\bar{c}_{1}}{p_{0}}\left(\psi(t)-\psi^{\circ}(t / t-1)\right)+\ldots+\frac{\bar{c}_{m}}{p_{0}}\left(\psi(t+1-m)-\psi^{\circ}(t+1-m / t-m)\right) \\
f^{\prime}(t+2)= & -\alpha_{1} f^{\prime}(t+1)-\alpha_{2} \psi(t)-\ldots-\alpha_{n_{a}+1} \psi\left(t+1-n_{a}\right) \\
& +z^{2}\left[\beta\left(z^{-1}\right)-\beta_{0}-\beta_{1} z^{-1}\right] \Delta u(t-1)+\frac{\bar{c}_{2}}{p_{0}}\left(\psi(t)-\psi^{\circ}(t / t-1)\right) \\
& +\ldots+\frac{\bar{c}_{m}}{p_{0}}\left(\psi(t+2-m)-\psi^{\circ}(t+2-m / t+1-m)\right)
\end{aligned}
$$

etc. Thus $\psi^{\circ}$ can be written in vector form as

$$
\psi^{\circ}=\mathbf{G}^{\prime} \mathbf{u}+\mathbf{f}^{\prime}
$$


where

$$
\begin{aligned}
\mathbf{f}^{\prime} & =\left[f^{\prime}(t+1), \ldots, f^{\prime}(t+N)\right]^{\mathrm{T}} \\
\mathbf{G}^{\prime} & =\left[\begin{array}{cccc}
g_{0}^{\prime} & & \\
g_{1}^{\prime} & g_{0}^{\prime} & & \\
\ldots & & & \\
g_{N-1}^{\prime} & g_{N-2}^{\prime} & \ldots & g_{0}^{\prime}
\end{array}\right]
\end{aligned}
$$

and where $g_{j}^{\prime}$ has the following recursive form

$$
g_{j}^{\prime}=\beta_{j}-\sum_{i=1}^{x} \alpha_{i} g_{j-i}^{\prime}
$$

where $x=\min \left(n_{a}+1, j\right)$ and $g_{0}^{\prime}=\beta_{0}=b_{0} p_{0}$.

It is easy to see that $g_{j}=g_{j}^{\prime}$ and $f(t+j)=f^{\prime}(t+j)(j=1,2, \ldots, N)$, which means that $\mathbf{G}=\mathbf{G}^{\prime}$ and $\mathbf{f}=\mathbf{f}^{\prime}$. Then (2.9) can be replaced by (4.7) and the recursive calculation of equations (2.3), (2.4) and (2.6) are not needed. The calculational load of the algorithm is reduced in this way. A comparison example between the calculation of $\psi^{\circ}$ in section 2 and the simplified version suggested above is given in the following table. We see that the number of operations is significantly decreased when the simplified version is used. We have here, for the sake of simplicity, put $P=Q=1, N=10$ and $n_{a}=n_{b}=n_{c}=n$. The abbreviations 'add' and 'mult' represent addition operation and multiplication operation respectively.

\begin{tabular}{lcccc}
\hline & $\mathrm{n}=1$ & $\mathrm{n}=2$ & $\mathrm{n}=3$ & $\mathrm{n}=4$ \\
\hline add (not simplified) & 183 & 301 & 437 & 602 \\
add (simplified) & 141 & 165 & 199 & 231 \\
mult (not simplified) & 231 & 345 & 477 & 630 \\
mult (simplified) & 137 & 153 & 174 & 198 \\
\hline
\end{tabular}

In the derivation above we have assumed that the plant's parameters are known. When the parameters are unknown, (2.1) is written as

$$
y(t)=y(t-1)+\theta^{\mathrm{T}} X(t-1)+\omega(t)
$$

where

$$
\begin{aligned}
X(t-1)= & {\left[-\Delta y(t-1), \ldots,-\Delta y\left(t-n_{a}\right),\right.} \\
& \left.\Delta u(t-1), \ldots, \Delta u\left(t-n_{b}-1\right), \omega(t-1), \ldots, \omega\left(t-n_{c}\right)\right]^{\mathrm{T}} \\
\theta= & {\left[a_{1}, \ldots, a_{n_{a}}, \ldots, b_{0}, \ldots, b_{n_{b}}, c_{1}, \ldots, c_{n_{c}}\right]^{\mathrm{T}} }
\end{aligned}
$$

Define $\hat{\theta}(t)$ and $\hat{\omega}(t)$ to be the estimates of $\theta$ and $\omega(t)$ at time $t$ respectively, viz.

$$
\begin{gathered}
\hat{\theta}(t)=\left[\hat{a}_{1}(t), \ldots, \hat{a}_{n_{a}}(t), \hat{b}_{0}(t), \ldots, \hat{b}_{n_{b}}(t), \hat{c}_{1}(t), \ldots, \hat{c}_{n_{c}}(t)\right]^{\mathrm{T}} \\
\hat{\omega}(t)=y(t)-y(t-1)-\theta(t-1)^{\mathrm{T}} \hat{X}(t-1)
\end{gathered}
$$

where

$$
\begin{aligned}
\hat{X}(t-1)= & {\left[-\Delta y(t-1), \ldots,-\Delta y\left(t-n_{a}\right),\right.} \\
& \left.\Delta u(t-1), \ldots, \Delta u\left(t-n_{b}-1\right), \hat{\omega}(t-1), \ldots, \hat{\omega}\left(t-n_{c}\right)\right]^{\mathrm{T}}
\end{aligned}
$$


The extended least squares method or other methods can be used to estimate $\hat{\theta}(t)$ and $\hat{\omega}(t)$. These estimates are then used to compute the control $u(t)$. The steps in this WGPC adaptive algorithm are shown in what follows.

Data: Given polynomials $P$ and $Q$, horizon $N$ and $N_{u}$.

Step 1. Estimate $A, B, C$ and $\omega(t)$;

Step 2. Compute $\mathbf{G}$ and $\mathbf{f}$ using (4.8)-(4.10);

Step 3. Compute the first row of matrix $\left(\mathbf{G}^{\mathrm{T}} \mathbf{G}+\overline{\mathbf{Q}}^{\mathrm{T}} \mathbf{Q}\right)^{-1}$.

Step 4. Compute $u(t)$ using (2.18);

Step 5 . Increase $t$ by one and return to the step 1 .

\section{Simulation}

An example is given in this section to show the performance of the simplified WGPC adaptive algorithm proposed in this paper. The plant to be controlled is described by

$$
\left(1-1 \cdot 7 z^{-1}+0.9 z^{-2}\right) y(t)=\left(1+2 z^{-1}\right) u(t-1)+\left(1+0.3 z^{-1}\right) \omega(t) / \Delta
$$

where $\omega(t)$ is a zero-mean white noise with variance 0.07 . In the adaptive algorithm we choose $P\left(z^{-1}\right)=0 \cdot 9+0 \cdot 1 z^{-1}, Q\left(z^{-1}\right)=0 \cdot 5+0 \cdot 6 z^{-1}, N=3, N_{u}=1$. The initial values of parameter estimates are $\hat{\theta}(0)=[-1,1 \cdot 2,0 \cdot 7,2 \cdot 3,0]^{\mathrm{T}}$.

Figure 1 shows the behaviour of $y(t)$ when it is tracking reference sequence $y_{r}(t)$. Figure 2 shows the corresponding control signal $u(t)$. It is seen that $y(t)$ tracks $y_{r}(t)$ quite well but there is a large overshoot in the beginning due to the effect of initial values of $\theta(0)$. Figure 3 shows the parameters' estimates of $\theta(t)$, which demonstrates the good convergence property of the parameter estimator.

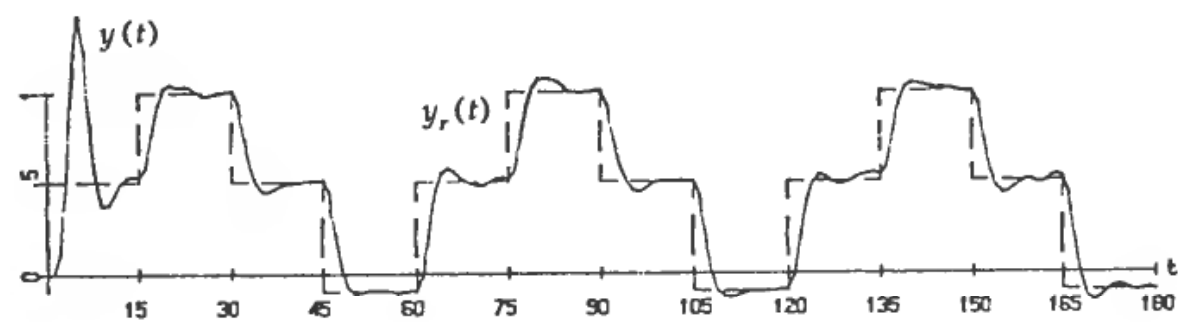

Figure 1. The reference sequence $y_{r}(t)$ and output $y(t)$.

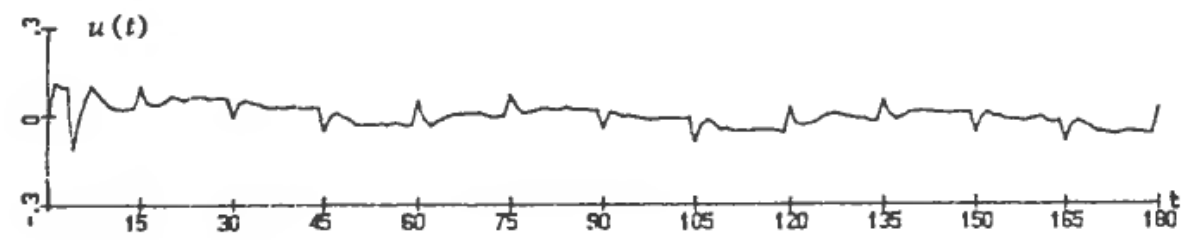

Figure 2. The corresponding control $u(t)$. 


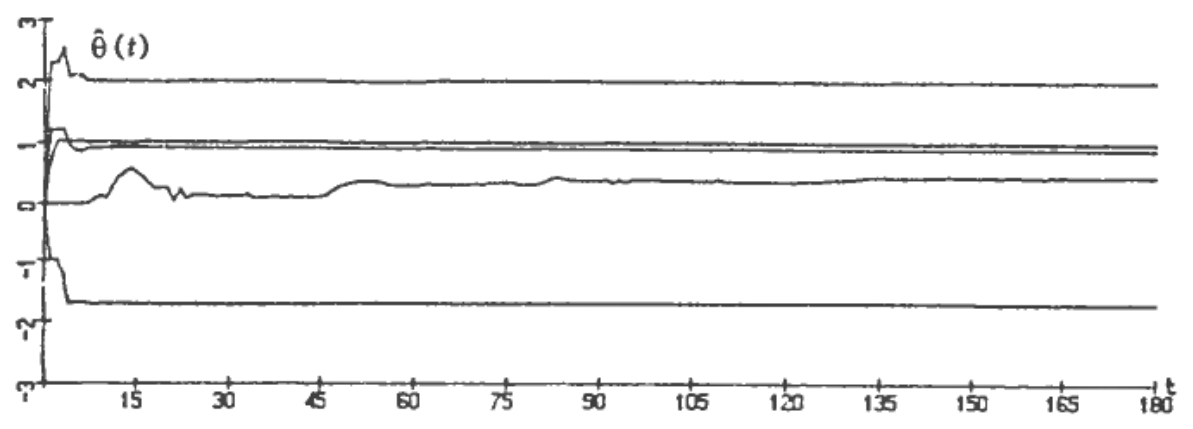

Figure 3. The parameter estimates.

\section{Conclusions}

A weighted generalized predictive controller has been derived in this paper. A concatenation property of the optimal prediction has been proved by which a simplified WGPC adaptive algorithm was developed. The simplified algorithm lowers significantly the number of calculational operations required without any significant change of the performance. This is believed to be of great importance in industrial control applications.

\section{ACKNOWLEDGMENTS}

The first author's work was supported by the Royal Norwegian Council for Scientific and Industrial Research (NTNF) and the National Natural Science Foundation of P.R. China.

\section{REFERENCES}

Clarke, D. W., and Gawthrop, P. J. (1979). Self-tuning control. IEE Proc. D., 126, 633-640. Clarke, D. W., Mohtadi, C. and TufFs, P. S. (1987a). Generalized predictive control, Part 1. Basic algorithm. Automatica, 23, 137-148.

Clarke, D. W., Mohtadi, C., and TuFfS, P. S. (1987b). Generalized predictive control, Part 2. Extensions and interpretations. Automatica, 23, 149-162.

Clarke, D. W. and Mohtadi, C. (1989). Properties of generalized predictive control. Automatica, 25, 859-875.

De Keyser, R. M. C., VAN De Velde, G. A. and Dumortier, F. A. G. (1988). A comparative study of self-adaptive long-range predictive control methods. Automatica, 24, 149-163.

De Keyser, R. M. C. and Van Cauwenberhe, A. R. (1982). Simple self-tuning multistep predictors. Preprints of the 6th IFAC Symp. on Identification and System Parameter Estimation, vol. 2, pp. 1558-1563.

FAVIER, G. (1987). Self-tuning long-range predictive controllers. Preprints of the 10th IF AC World Congress, vol. 10, pp. 80-87.

Garcia, C. E., Prett, D. M. and Morari, M. (1989). Model predictive control. Theory and practice-a survey. Automatica, 25, 335-348.

Goodwin, G. C. and Sin, K. S. (1984). Adaptive filtering, prediction and control (Prentice-Hall, Inc., Englewood Cliffs). 